\title{
Becoming in touch with industrial robots through ethnography
}

\author{
Ned Barker ${ }^{\dagger}$ \\ Institute of Education \\ University College London \\ United Kingdom \\ edmund.barker@ucl.ac.uk
}

\author{
Carey Jewitt \\ Institute of Education \\ University College London \\ United Kingdom \\ c.jewitt@ucl.ac.uk
}

\author{
Sara Price \\ Institute of Education \\ University College London \\ United Kingdom \\ sara.price@ucl.ac.uk
}

\begin{abstract}
Touch is central to communication and social interaction. For both humans and robots touch is a mode through which they sense the world. A second wave of industrial robots is reshaping how touch operates within the labor process. Recent studies have turned their attention to the role of touch in Human-Robot Interaction (HRI). While these studies have produced useful knowledge in relation to the affective capacities of robotic touch, methods remain restrictive. This paper contributes to expanding research methods for the study of robotic touch. It reports on the design of an ongoing ethnography that forms part of the InTouch project. The interdisciplinary project takes forward a socially orientated stance and is concerned with how technologies shape the semiotic and sensory dimensions of touch in the 'real world'. We contend that these dimensions are key factors in shaping how humans and robots interact, yet are currently overlooked in the HRI community. This multi-sited sensory ethnography research has been designed to explore the social implications of robotic touch within industrial settings (e.g. manufacturing and construction).
\end{abstract}

\section{CCS CONCEPTS}

- Robotics $\sim$ Social and Behavioural Sciences $\sim$ Haptics

\section{KEYWORDS}

Robotic touch; Multi-sited sensory ethnography; Industrial robots

\section{Introduction}

1.1. HRI Touch. The embodiment of robots makes physical contact and touch possible [1]. The possibilities for robotic touch are expanding within industrial setting as a consequence of technological developments. Robots are developing more sophisticated tactile capabilities affording them new roles in the workplace as they take over "dirty, dull and dangerous work" (p.563) [2]. At the same time the design of robots (e.g. Cobots and Robotic Exoskeletons) is fostering closer collaborations with human workers. Wearable robotics in construction and manufacturing, for example, are transforming laborers' ability and stamina to touch/move heavy objects. On one level, these

Permission to make digital or hard copies of part or all of this work for personal or classroom use is granted without fee provided that copies are not made or distributed for profit or commercial advantage and that copies bear this notice and the full citation on the first page. Copyrights for third-party components of this work must be honored. For all other uses, contact the Owner/Author.

HRI '20 Companion, March 23-26, 2020, Cambridge, United Kingdom.

(C) 2020 Copyright is held by the owner/author(s).

ACM ISBN 978-1-4503-7057-8/20/03

DOI: https://doi.org/10.1145/XXXXXXX.XXXXXXX developments represent a change in touch practices as physical and functional events - to be analyzed in terms of efficiency, ergonomics, energy expenditure and so on. On another, emerging industrial robots can be understood as new mediators and producers of the social landscapes where manual labor occurs because they reshape how touch operates within a setting or process. Consequently, industrial robots have a social role within and across industrial sites. To date, however, the social character of robotic touch has been largely neglected in industrial robotics.

1.2. HRI Methods for Exploring Touch. Current HRI methods are designed to measure how robotic touch affects humans in predefined interactions and contexts, drawing on positivist epistemologies to collect quantitative forms of data. Broadly speaking, two types of methods are utilized. First, participant surveys are used to gain quantified measurements of how robotic touch affected them after controlled interactions with robots e.g. [3-5]. Second, these data are often triangulated with highly structured observations of robotic touch encounters that take place within similarly controlled environments e.g. [4]. While valuable knowledge has been generated with these methods, contributions have yet to consider the sociality of robotic touch (in the broadest sense) in the 'real world' [6] with real people.

We turn to ethnography of robotic touch to speak to this methodological gap, bringing the semiotic and sensory dimensions of touch between humans and robots to the fore. Situated across five sites, our ethnography of robotic touch seeks to contribute to extending the use of ethnography in HRI by providing important insight into how new robotic technologies reshape touch practices and their wider social implications.

1.3. Ethnography. Defining features of Ethnography include the researcher participating in, and observing, social (and sensory) encounters that take place in the 'real world'; and data collection methods rely on making fieldnotes that interpret and record what unfolds during participant observation. Observing how robotic touch operates socially outside of lab settings has been identified as area for future research [4] and the potential of ethnography for HRI has been signaled. Although unresolved differences within HRI remain in terms of what ethnographies (should) involve, bringing together empirical and methodological contributions, the Special Issue on Ethnography in HRI Research [7] recognized its methodological potential to develop contextually rich and dynamic accounts of HRI beyond controlled environments. Nonetheless, ethnography remains "more or less absent from the field of human robot interaction" (p.180) [7] and there are no ethnographic accounts of robotic touch in the 'real world'. We argue ethnography provides a vehicle to deepen HRI understanding of the sensory and semiotic dimensions of robotic touch. To illustrate the potentials of ethnography for studying the sociality of robotic touch in 'real world' industrial settings, this paper sets out our research design. 
First, we position our approach against methodological tensions within HRI [7]-[9], utilizing ethnography to specifically overcome limitations within HRI's insights into robotic touch. Second, we unpack the multi-sited and sensory aspects of our design with reference to the specificities of robotic touch in industrial settings.

\section{METHODS: ETHNOGRAPHY AND HRI}

The vision of ethnography outlined below responds to the specificities of studying the semiotic and sensory dimensions of robotic touch in industrial settings our ongoing research embraces both multi-sited and sensory strands of ethnography.

2.1. Reflexivity in Ethnography. Reflexivity, central to an ethnographic approach, is a process through which a researcher reflects on the data collection and interpretation processes to become aware of and to articulate, their multiple roles in the construction of knowledge (i.e. how their analytic focus and presence shapes their relationship to the field of study and the data). It has been suggested that there is a lack of, and a need for, ethnography in HRI [9] that more actively embraces reflexivity. For Chun [9], reflexive approaches require an appreciation of the subjective and interpretive processes of knowledge creation, thereby distancing ethnography from positivism, HRI's dominant epistemological frame. To do this, we maintain a reflexive journal. Entries have reflected on: rapport; positionalities; use of theory; and the ways in which embodied experiences frame interpretation and analysis. These processes enable us to acknowledge our situated-ness, and our "co-construction" of our own data through our "embodied participation in the empirical field" [8] (p.120). These reflections stimulate critical interdisciplinary discussions as we consider the sociality of robotic touch through semiotic and sensory lenses [10].

2.2. Ethnographic Participation. Observations alongside questionnaires are the primary method in HRI to capture the affective capacities of robotic touch (see 1.2). This generates narrow and numerical representations of robotic touch, limited further by decontextualizing the sociality of touch by removing it from 'real world' settings and providing "an observation of touch, not a full participation in touching or being touched" [4]. Current ethnography in HRI, has exclusively relied upon a 'fly on the wall' technique [9], and the active participation of the ethnographer is muted. Without an emphasis on participatory and reflexive approaches to ethnography, HRI tends to reduce fieldwork towards a generic form of qualitative observation, thereby undercutting ethnography's value to develop contextually rich and dynamic accounts of the sensory and semiotic dimensions of 'real world' robotic touch [6]. To participate is to touch and be touched and is therefore an important resource in bringing the ethnographer closer to sensory and semiotic action.

In contrast, the InTouch design foregrounds the importance of reflexivity and participation. Becoming 'in touch' with robots within, and across, environments is being pursued through participatory approaches, such as: attending training, laboring alongside workers and robots, directly experiencing robotic touch, and conversations with participants during an activity.

2.3. Multi-sited Ethnography and Robotic Touch. Technological innovations that characterize the second wave of robotics (the ability to perform tasks with greater autonomy, sensing, and dexterity) afford industrial robots to move into new environments and take over more touch tasks. Against this backdrop robotic touch, as it appears in 'the real world', cannot be thought of or researched as a homogenous phenomenon. Therefore, our object of study consists of a set of highly differentiated events that are 'spatially disperse'. This lends itself to a multi-sited approach [11]. With a view to describe how touch is being transformed in 'real world' industrial settings, through new human-robot working relationships, five sites were selected. Site 1: We are exploring touch on construction sites where robotic technologies are being used for in-situ fabrication assisting builders in unstructured environments. Site 2: Cobots are suited to close proximity work with humans, and at another site we consider close-quarter touch interactions in a manufacturing context. Site 3: We are seeking to understand sensory and semiotic implications of when dirty touch tasks are outsourced to sorting robots in a waste management center. Site 4: The use of wearable robotics on a construction provides opportunity to study touch in these intimate human-robot partnerships. Site 5: we are spending time with a roboticists company that are envisioning, and planning for, the future of robotic touch in industry. Immersing ourselves in these 'real world' contexts will enable us to explore current varied expressions of robotic touch, its future directions and social impacts.

2.4. Sensory Ethnography and Robotic Touch. The InTouch project has drawn upon sensory ethnography to explore the sensory and semiotic dimensions of the tactile as it is digitally mediated in the real world [6], [10]. Using this approach [12] we develop ethnographic sensitivities capable of depicting sensory landscapes, giving the 'real world' its feel, and to deepen our accounts of robotic touch. Reflexive and participatory approaches fold into our sensory sensitivities to craft thick descriptions of robotic touch in the 'real world'. This enables us to: illustrate the social implications of industrial robots; draw on interdisciplinary expertise to generate novel knowledge of robotic touch in the 'real world'; and extend conceptualization of robotic touch beyond its affective qualities by bringing semiotic and sensory dimensions of touch to the fore.

\section{CONCLUSION}

The design of the InTouch projects' ethnography of robotic touch speaks to a significant gap in HRI accounts of robotic touch by bringing to the fore the semiotic and sensory dimensions of touch between humans and robots. A multi-sited sensory ethnography will generate contextually rich and dynamic empirical accounts of robotic touch, that attend to the social qualities of digitally mediated touch, an account currently absent from the HRI literature. Our research will also contribute conceptual and methodological understanding of robotic touch. In summary, our use of ethnography will inform the study of robotic touch in four key ways: 1) Exploring the social implications of robotic touch in 'real world' working environments; 2) Detailed description of how touch is being reshaped as robots are tasked with new jobs, and take part in new collaborations; 3) Providing insight on how workers relate to, think about, articulate and practice touch differently as a result of new robotic technologies entering their workplace; and 4) Developing methodological approaches and conceptual apparatus for researching robotic touch. The poster presentation will share some preliminary data and articulate how ethnography has enabled us to contribute to understanding robotic touch in the context of HRI.

\section{ACKNOWLEDGMENTS}

This research is funded as a part of the IN-TOUCH project, a European Research Council Consolidator Award (Award Number: 681489). 
Becoming in touch with industrial robots through ethnography

\section{REFERENCES}

[1] H. Cramer, N. Kemper, A. Amin, and V. Evers, "The effects of robot touch and proactive behaviour on perceptions of human-robot interactions," Proc. 4th ACM/IEEE Int. Conf. Human-Robot Interact. HRI'09, pp. 275276, 2009.

[2] L. Royakkers and R. van Est, "A Literature Review on New Robotics: Automation from Love to War," Int. J. Soc. Robot., vol. 7, no. 5, pp. 549$570,2015$.

[3] S. Yohanan and K. E. MacLean, "The Role of Affective Touch in HumanRobot Interaction: Human Intent and Expectations in Touching the Haptic Creature," Int. J. Soc. Robot., vol. 4, no. 2, pp. 163-180, 2012.

[4] T. Arnold and M. Scheutz, "Observing Robot Touch in Context: How Does Touch and Attitude Affect Perceptions of a Robot's Social Qualities?," ACM/IEEE Int. Conf. Human-Robot Interact., pp. 352-360, 2018.

[5] T. L. Chen, C. H. A. King, A. L. Thomaz, and C. C. Kemp, "An Investigation of Responses to Robot-Initiated Touch in a Nursing Context," Int. J. Soc. Robot., vol. 6, no. 1, pp. 141-161, 2014.

[6] C. Jewitt, S. Price, K. Mackley, N. Giannoutsou, and D. Atkinson,
HRI '20 Companion, March 23-26, 2020, Cambridge, UK.

Interdisciplinary Insights for Digital Touch Communication. Springer International Publishing, 2020

[7] C. Hasse, S. Trentemøller, and J. Sorenson, "Special Issue on Ethnography in Human-Robot Interaction Research," J. Behav. Robot., vol. 10, no. 1, pp. 180-181, 2019.

[8] L. Blond, "Studying robots outside the lab: HRI as ethnography," J. Behav. Robot., vol. 10, no. 1, pp. 117-127, 2019.

[9] B. Chun, "Doing autoethnography of social robots: Ethnographic reflexivity in HRI," J. Behav. Robot., vol. 10, no. 1, pp. 228-236, 2019.

[10] C. Jewitt and K. Leder Mackley, "Methodological dialogues across multimodality and sensory ethnography: digital touch communication," Qual. Res., vol. 19, no. 1, pp. 90-110, 2019.

[11] M. Falzon, Multi-sited Ethnography: Theory, Praxis and Locality in Contemporary Research. Ashgate: Ashgate Publishing Company, 2016.

[12] S. Pink, Doing Sensory Ethnography, 2nd ed. London: SAGE Publications Ltd, 2015. 